\title{
Germanica
}

\section{La question des « Mischehen » sous le Troisième Reich et l'exemple des Klemperer}

Eine " Mischehe " im Dritten Reich. Eva und Victor Klemperer

\section{Didier Herlem}

\section{OpenEdition}

\section{Journals}

Édition électronique

URL : http://journals.openedition.org/germanica/2457

DOI : $10.4000 /$ germanica. 2457

ISSN : 2107-0784

\section{Éditeur}

Université de Lille

\section{Édition imprimée}

Date de publication : 30 décembre 2000

Pagination : 23-39

ISBN : 9782913857094

ISSN : 0984-2632

\section{Référence électronique}

Didier Herlem, «La question des « Mischehen » sous le Troisième Reich et l'exemple des Klemperer », Germanica [En ligne], 27 | 2000, mis en ligne le 01 avril 2014, consulté le 06 octobre 2020. URL : http:// journals.openedition.org/germanica/2457 ; DOI : https://doi.org/10.4000/germanica.2457

Ce document a été généré automatiquement le 6 octobre 2020.

(ㄷ) Tous droits réservés 


\title{
La question des « Mischehen » sous le Troisième Reich et l'exemple des Klemperer
}

\author{
Eine « Mischehe » im Dritten Reich. Eva und Victor Klemperer
}

Didier Herlem

1 En guise de préface à LTI, Victor Klemperer consacre quelques pages à l'analyse du concept d'héroïsme. Partant du constat que comme tant d'autres ce concept a été "souillé ", " empoisonné " par le national-socialisme, que des générations entières, hommes et femmes, jeunes et moins jeunes s'y sont laissés "totalement piéger », il oppose à la notion d'héroïsme imposée par le Troisième Reich, selon lui «la plus douteuse qui soit ", un autre héroïsme.

J'ai connaissance d'un autre héroïsme, bien plus désespéré, bien plus discret, d'un héroïsme privé de tout soutien lié à l'appartenance, à une armée, à un groupe politique, privé du moindre espoir d'une gloire future, d'un héroïsme totalement réduit à ses propres moyens. Celui de ces quelques épouses aryennes (elles n'ont guère été nombreuses), qui ont résisté à toutes les pressions exercées pour qu'elles se séparent de leurs maris juifs ${ }^{1}$.

2 Suit alors un hommage lyrique à ces femmes, à leur abnégation, leur vouloir-vivre, leur autodiscipline, leur force de caractère, leur stoïcisme, que l'auteur clôt par cette formule: «cet héroïsme transcende tous les autres» («Das ist Heroismus über jeglichem Heldentum $»)^{2}$.

3 Cet hommage n'a rien d'abstrait. Il peut même être considéré comme un fidèle résumé du calvaire enduré par ces femmes : menaces, coups, crachats, injures de la part de la Gestapo ; privations alimentaires; maladie, décès, tentation du suicide, synonymes de mort pour le survivant; environnement morbide des "Judenhäuser» (maisons réservées aux juifs); conditions matérielles de vie répugnantes, se dégradant sans cesse ; solitude et promiscuité ; enfin et surtout, confrontation permanente à l'angoisse désespérée, désespérante, de leur compagnon. 
à travers ces lignes, les Carnets ${ }^{3}$ en apportent la preuve flagrante -, c'est sa femme, Eva, dont il trace le portrait, c'est elle qu'il honore.

Mariés depuis le 29 juin 1904, Victor et Eva Klemperer mènent jusqu'à l'arrivée au pouvoir des nazis l'existence d'un couple « sans histoire » confronté comme les autres aux seuls aléas de la vie. Ils forment un couple uni. L'un et l'autre sont chrétiens. D'origine juive, Victor se sent allemand, rien qu'allemand, il est nationaliste et patriote $^{4}$. Eva revendique volontiers la spécificité de son ascendance scandinave, alors que ses ancêtres se sont installés en Allemagne durant la guerre de Trente ans. L'arrivée au pouvoir des nazis et la politique de discrimination raciale qui s'ensuit va soudain transformer leur union en une entité hybride et condamnable: une « Mischehe ». Le terme préexistait et existe toujours, puisqu'il sert à désigner un couple de religion ou de confession différente, en particulier pour l'Allemagne, l'alliance d'un catholique et d'un protestant. L'antisémitisme nazi lui conféra un sens nouveau. Il qualifie l'union d'un aryen avec une juive, ou l'inverse. Il faut donc l'entendre au sens de « mariage racialement mixte ». Dans la même logique, les enfants issus de ces unions seront désormais qualifiés de Mischlinge : "métissés de sang juif et aryen ». Au nom de quoi ? Du projet racialiste nazi. Peut être n'est-il pas inutile de rappeler que ce projet avait sa cohérence. S'inspirant très largement du darwinisme social, il partait de l'idée que le moteur de l'Histoire n'était pas la lutte des classes, mais la lutte des nations définies en l'occurrence comme des entités raciales. Cette lutte devait conduire à la sélection de la race la meilleure, à qui reviendrait le droit de dominer le monde. Dans cette perspective, la priorité du politique devait être de restaurer l'homogénéité du " corps social », de le débarrasser de tous les éléments étrangers nuisibles, inférieurs, dégénérés, parasitaires. L'objectif est de refonder la nation sur des bases biologiques, d'instaurer un régime nouveau, celui de la "biocratie totalitaire ». Seul un tel régime permettrait de transformer le «corps social», redevenu "pur et sain », en un instrument efficace de conquête militaire, une arme invincible dans la lutte pour l'hégémonie.

Outre le darwinisme social, le projet racialiste nazi reprit à son compte la tradition antisémite qu'il radicalisa à outrance. Le juif devint ainsi l'archétype de l'élément nuisible, inférieur, dégénéré, parasitaire, qui idéologiquement et biologiquement voulait la ruine du peuple allemand.

L'arrivée au pouvoir des nazis en 1933 signifia non seulement le début d'une lutte ouverte contre tout ce qui était idéologiquement assimilable au juif (marxisme, libéralisme, féminisme, individualisme, démocratie, etc...), mais aussi pour l'ensemble des citoyens allemands l'instauration arbitraire d'un nouveau principe de division élémentaire du champ social : l'appartenance raciale. Dans son application politique et pratique, ce principe va séparer progressivement «juifs » et «non juifs ». En quatre étapes d'une escalade quasi continue, Allemands et Allemandes d'origine juive furent soumis à un processus d'isolement, de dissimilation, d'expropriation, de paupérisation, de destruction par le travail et d'extermination qui déboucha sur la « solution finale » de sinistre mémoire. Au cours de ce processus qui s'étalera sur les douze années que dura le régime, se mettra en place une implacable dialectique de déshumanisation du bourreau tentant par tous les moyens de déshumaniser sa victime. Le seul moyen de passer à l'acte et d'accomplir des actions aussi barbares résidait dans la 
déshumanisation de l'autre, dans sa "fécalisation " pour reprendre une expression de $\mathrm{J}$. Chasseguet-Smirgel ${ }^{5}$, comme mode de phagocytation et préalable à l'expulsion.

8 Isolement et dissimilation ne pouvaient s'obtenir qu'en tranchant dans le vif de la communauté allemande toute entière. Cette entreprise de lacération du tissu social au sens proprement chirurgical du terme va provoquer une déchirure entre les citoyens, les communautés religieuses, les familles, devant laquelle le pouvoir ne recula guère.

9 Restait un cas, celui des couples «racialement mixtes» et de leur éventuelle progéniture, les « Mischlinge » où l'acte chirurgical ne pouvait être mené si aisément à bien.

10 Quelle fut en fait la politique nazie à l'égard de ces couples? Comment ces couples réagirent-ils aux effets de cette politique? Comment en particulier, Victor et Eva Klemperer vécurent-ils ces douze années?

11 C'est à ces questions que la présente étude, à partir de l'analyse des Carnets et en privilégiant délibérément la dimension Mischehe, veut tenter de répondre.

12 La politique nazie à l'égard des Mischehen et des Mischlinge ne fut pas déterminée d'emblée de façon claire. Le premier signe identifiable fut donné lors du premier boycott total des magasins juifs organisé par les nazis fin mars début avril 1933. À cette occasion, l'on procéda au recensement des établissements commerciaux dont le propriétaire était juif ou marié à un juif. Klemperer note d'ailleurs cette singularité le 31 mars 1933 :

La religion importe peu, seule compte la race. Si des propriétaires du magasin l'homme est juif, la femme chrétienne ou l'inverse: le magasin est considéré comme juif ${ }^{6}$.

Le premier décret d'application de la loi sur le rétablissement du fonctionnariat professionnel paru le 11 avril 1933, précise, quant à lui, la notion floue de "non aryen ». Il l'interprète de façon extensive en y adjoignant les Mischlinge, les métissés de sang juif et aryen, « au premier et au second degré » (Mischlinge ersten und zweiten Grades) ${ }^{7}$. Le 5 juillet 1933, un décret d'application du Reinhardt Programm, la loi organisant la lutte contre le chômage, exclut des prêts aux jeunes ménages tous les couples non aryens, mais aussi ceux dont un seul des futurs époux ne serait pas de "pur sang allemand». Le 4 octobre 1933, enfin, la loi sur les rédacteurs en chef (Schriftleitergesetz) précise que pour être rédacteur, il fallait faire la preuve de son origine "aryenne", mais aussi, au cas où l'on était marié, que l'épouse remplisse également cette condition ${ }^{8}$. Plus généralement, il était clair que désormais tout Allemand qui épousait une "non aryenne » (ou l'inverse) perdait ipso facto la capacité à devenir fonctionnaire 9 . Dès 1933 également, on peut noter des tentatives de criminalisation sauvage des individus accusés de transgresser le nouvel ordre sexuel et racial. Les fautifs, pour lesquels on invente le délit de Rassenschande, de "souillure raciale ", sont livrés à la vindicte publique. Les termes de Judensau (truie à juifs), de Judenweib (femelle à juifs), Judenhure (fille à juifs) sont systématiquement employés, banalisés. Cette campagne contre les Rasseschänder fut constante durant les premières années du Troisième Reich. Elle reçut au demeurant le renfort de publications à caractère scientifique. Ainsi le magazine médical Santé du peuple allemand (Deutsche 
Volksgesundheit) aborde-t-il dans sa livraison de janvier 1935 la question des conséquences engendrées par la pollution d'une " aryenne » par un « juif » :

La semence d'un homme d'une autre race est un albumen étranger à l'espèce. Lors de l'accouplement, la semence masculine est aspirée partiellement ou en totalité par la matrice féminine et passe ainsi dans le sang. Un seul coït avec un juif suffit, chez une femme aryenne, à empoisonner son sang pour toujours. Avec l'albumen étranger à l'espèce, elle a également absorbé l'âme étrangère. Même si elle épouse un homme aryen, jamais plus elle ne pourra avoir d'enfants aryens de race pure, elle n'aura que des bâtards ${ }^{10}$.

14 Cette campagne destinée à préparer l'opinion aux lois de Nuremberg, fut accompagnée de nombreux procès spectaculaires pour viol, pour "amputation de la race» (Entrassung), des procès fabriqués de toute pièce, mais qui permirent d'éliminer de présumés Rasseschänder. elle permet de saisir ce que Mischehen et Mischlinge pouvait représenter pour les fanatiques du national-socialisme, à savoir, d'une part un crime contre le sang, mais un crime légalisé, et d'autre part, les fruits vivants, légitimes d'un tel crime.

15 La première des lois de Nuremberg, dite Reischbürgergesetz, scinde la communauté allemande, et par conséquent les couples «mixtes » en deux entités distinctes. D'un côté, les citoyens du Reich de sang allemand ou racialement apparenté, lesquels sont seuls «détenteurs des droits politiques dans leur totalité». De l'autre, les « ressortissants de l'État allemand» (Staatsangehörige) privés de tout droit politique. le premier décret d'application (14 novembre 1935) définit pour la première fois ce qu'est un juif pour un nazi et également ce qu'est un Mischling: s'il est membre de la communauté religieuse juive, il est « ressortissant de l'État », s'il n'en est pas membre, il est « citoyen du Reich ».

La seconde loi de Nuremberg (Gesetz zum Schutze des deutschen Blutes und der deutschen Ehre) interdit les mariages entre juifs et non juifs. Les rapports sexuels entre eux sont officiellement criminalisés et assortis de lourdes peines. Les mariages entre juifs et Mischlinge au second degré sont également interdits.

17 Le processus de dissimilation se poursuit par l'introduction en 1938 d'une carte d'identité spéciale frappée d'un «J", par le port obligatoire de l'étoile jaune, par l'exclusion des juifs de la vie économique et l'organisation du pillage légal de leurs biens. L'«aryanisation » de la propriété foncière décrétée le 28 décembre 1938 offre la particularité de s'étendre aux biens possédés par le conjoint «non juif » des couples mixtes : le mariage "judaïse » aussi la propriété aryenne, en fonction d'une logique dont la seule finalité est de donner au pillage le maximum d'ampleur ${ }^{11}$. Il semble cependant que, dans ce domaine, si l'on en croit les témoignages des Carnets, l'atteinte au droit de propriété n'a pas été systématiquement pratiqué.

Le cas des Mischlinge et des Mischehen semble n’avoir été abordé sur un mode global que tardivement par les exterminateurs nazis.

19 Les Mischlinge, qui avaient été enregistrés au même titre que les «juifs » dans les fichiers de la Gestapo, furent exclus de la Wehrmacht le 20 avril 1940, alors que jusquelà, ils pouvaient être soldats et s'élever jusqu'au grade de caporal dans la hiérarchie militaire ${ }^{12}$. Lors de la conférence de Wannsee, le 20 janvier 1942, Heydrich proposa dans la perspective de la «solution finale » d'assimiler aux juifs les Mischlinge du premier degré. Le Secrétaire d'État auprès du Ministre de l'Intérieur, Stuckart, proposa pour sa part la stérilisation de tous les Mischlinge. Aucune décision définitive n'ayant été 
arrêtée, Eichmann organisa une conférence sur ce sujet en 1942. Résultat : interdiction totale de se marier, exclusion de l'école et de l'université. Mais on ne put se résoudre à fixer définitivement leur sort. À la suite d'une nouvelle conférence réunie le 27 octobre 1942, on décida de laisser aux Mischlinge du premier degré le choix entre stérilisation et déportation. On eut également recours de façon ponctuelle à l'euthanasie ${ }^{13}$. Le 13 octobre 1943, Göring décida de mettre un terme à ces atermoiements et opta pour la procédure dite d'élimination par le travail (Vernichtung durch Arbeit). Celle-ci entra aussitôt en application. Vers la fin de l'année 1944, les Mischlinge qui n'étaient pas encore en camp de travail commencèrent à être déportés au camp de Theresienstadt. La majorité d'entre eux, dont la seule tare était d'être des témoins vivants de la collusion honnie de deux races, disparut ainsi, soit par épuisement physique, soit par extermination directe. Ce qu'ils avaient en eux de « sang allemand » n'avait pas réussi à les protéger jusqu'au bout contre l'obsession nazie de la pureté raciale.

Quant aux couples mixtes, ils suscitèrent également bien des hésitations. l'un des conjoints étant aryen, il pouvait difficilement être traité exactement comme un « juif », mais en même temps, il constituait l'archétype du mauvais aryen, "oublieux de sa race » (Artvergessen), et à ce titre pouvait difficilement être totalement absout ${ }^{14}$. Lors de la conférence de Wannsee, Heydrich proposa que les Mischehen fassent l'objet d'un examen au cas par cas, tandis que Stuckart formula l'idée d'annuler automatiquement tous les mariages mixtes. La conférence organisée par Eichmann en mars 1942 avança une troisième solution : exercer le maximum de pression sur le conjoint « aryen " pour qu'il demande le divorce de son propre chef. À l'expiration d'un délai de réflexion donné et au cas où le conjoint " aryen » n'avait pas cédé, le Procureur serait habilité à engager lui-même - au nom de l'intérêt de la communauté du peuple allemand - une procédure de divorce forcé. Dans l'un comme dans l'autre cas, le divorce devait être prononcé avec une extrême rapidité. Cette dernière solution fut définitivement retenue lors de la seconde conférence du 27 octobre 1942. Un an plus tard, les nazis décidèrent d'opérer une distinction entre deux types de Mischehen : d'une part les couples mixtes dits " privilégiés » (un non aryen de confession chrétienne marié à un aryen) et d'autre part les couples mixtes dits " non privilégiés » (un non aryen de confession juive marié à un aryen). Si les premiers pouvaient être épargnés, surtout s'il y avait des enfants, les seconds devaient être détruits. En février 1943, à Berlin, la Gestapo lança une première opération contre eux. Une centaine d'hommes qui vivaient en «Mischehen », furent arrêtés. Selon Klaus Drobisch, cette opération fut un échec, car elle provoqua "sans doute la seule manifestation de rue contre la terreur de la Gestapo en Allemagne ${ }^{15}$. Les épouses se rassemblèrent en effet devant le bâtiment où étaient séquestrés leurs conjoints et exigèrent la libération immédiate de leurs maris. Elles eurent partiellement gain de cause, ce qui prouve à quel point était délicat le travail de dissimilation lorsqu'il fallait trancher un lien affectif et lorsque l'iniquité des mesures prises était flagrante. Après tout, ces couples s'étaient formés avant la nouvelle législation raciale. Ils n'avaient rien d'illicite. Ils illustraient jusqu'à la caricature l'arbitraire de la politique nazie, son absurdité intrinsèque, l'état de non droit où vivait l'Allemagne depuis 1933.

21 Après l'échec partiel de février 1943, la décision fut prise d'en finir avec les Mischehen. Solution retenue : « l'élimination par le travail ». Le 18 décembre 1943, Himmler voulut accélérer le processus par la déportation des conjoints « juifs ", dans tous les cas où le divorce avait été prononcé et où le conjoint aryen était décédé. Le 13 septembre 1944, il décida l'arrestation systématique des Mischehen « non privilégiés ». Ces couples furent 
dépossédés de leurs biens et séparés. Les conjoints "juifs » rejoignirent le camp de Theresienstadt, quant aux «non juifs », la Gestapo leur octroya un délai de deux jours pour quitter les lieux. Pour la seule année 1945, 1954 conjoints «non juifs» de Mischehen arrivèrent à Therensienstadt. Ce furent les derniers déportés, les dernières victimes des persécutions nazies ${ }^{16}$.

Les Carnets de Victor Klemperer apparaissent comme un observatoire privilégié de ce que fut l'existence de ces couples, de ce que furent leurs réactions face au processus de dissimilation dont ils furent les victimes. Le 9 juin 1942, il écrit :

Autrefois, j'aurais été mortifié de ne pas être un écrivain (Dichter), maintenant, je pense sans cesse au Curriculum, à l'art de porter témoignage : la femme aryenne, le couple mixte. Des types, des cas observés... ${ }^{17}$

Eva et Victor Klemperer mis à part et sauf omission de notre part, ce sont en tout cinquante sept Mischehen qui sont évoqués au fil des pages. Outre les différences de statut administratif (privilégiés ou non) tous ont en commun la confrontation avec le mécanisme sadique (" die sadistische Maschine geht eben über uns weg ", 9 décembre 1939) de dissimilation. Historiquement, peuvent être distinguées trois phases: la première va de 1933 à 1940, durant laquelle le conjoint « aryen » n'est pas directement frappé par des mesures discriminatoires, mais est contraint de vivre avec l'autre sa marginalisation (perte de l'emploi, mise en retraite anticipée, réduction du train de vie), partage son angoisse pour l'avenir, sa solitude. De 1940 à 1942, tout change avec la mise en place des quartiers et des maisons réservées (Judenviertel; Judenhaüser). L'illusion d'une vie normale disparaît. La promiscuité-solitude règne au sein d'une communauté factice que seuls l'adversaire commun, la précarité, l'angoisse de la déchéance et de la mort rapprochent, avec la tentation permanente pour les " aryens " de s'en prendre aux juifs, de les rendre responsables de la situation, alors qu'eux pourraient vivre "normalement » à condition de renier leur couple. À partir de 1942, donc après la conférence de Wannsee, s'ouvre une troisième et dernière phase où la sécurité assurée par la présence du conjoint «aryen » semble devenir de plus en plus aléatoire. Les arrestations arbitraires se multiplient, le danger d'une séparation, d'une déportation, l'imposition du travail obligatoire avec en outre les maladies de carence, les privations de toute sorte, rendent l'espérance d'une survie possible toujours plus illusoire. La hantise de la séparation et de la mort se fait omniprésente, et ce jusqu'à l'écroulement final du régime.

24 À travers le prisme des Carnets, le lecteur prend conscience de la nature exacte de l'épreuve subie par ces couples dont la seule et unique faute est de ne pas correspondre aux nouveaux critères sociaux imposés en toute illégalité par le régime. Un "crime " rétro-actif en quelque sorte. La première phase de la dissimilation se caractérise par la sensation de solitude, d'isolement, une quarantaine permanente, engendrée par la réduction des libertés, les réactions hostiles, l'angoisse des "Aryens » susceptibles de payer cher une trop grande "cordialité à l'égard des juifs» (Juden-Freundlichkeit) ${ }^{18}$. Les deux phases suivantes sont déterminées par l'univers du « Judenhaus ».

Ce « camp de concentration haut de gamme » («gehobenes $\mathrm{KZ} »)^{19}$, présente en effet des caractéristiques bien particulières. C'est un univers carcéral à géométrie variable, en fonction de l'escalade des interdits et du statuts des individus, un microcosme 
reposant uniquement sur l'existence de liens affectifs légalisés entre deux êtres et l'exercice sur eux de la violence du nouvel ordre (les visites de la Gestapo). Promiscuité, absence d'affinités interindividuelles, disparité des origines, des professions, des religions, des références culturelles, accentuent a priori l'altérité de l'autre, voire l'exacerbent. De plus, c'est un univers strictement hiérarchisé, et pas uniquement sur le plan symbolique. À chaque statut (juif / aryen, mais aussi privilégié / non privilégié, porteur de l'étoile / non porteur de l'étoile) correspond une différence de traitement plus ou moins considérable. Le Judenhaus correspond à une mise en quarantaine d'un groupe hétéroclite par rapport au reste de la société, en opposition de plus en plus radicale avec le reste de la société, au fur et à mesure qu'y progresse la dissimilation des citoyens d'origine juive. Ces îlots ont la particularité de regrouper sous la contrainte « juifs» et « aryens ». Le " respect» de l'union incarnée par chaque couple, puisqu'on ne les sépare pas par la force, et sous-tendu par la volonté de le pousser à l'autodestruction. Chaque conjoint aryen dispose en permanence de la clé lui permettant d'échapper à cet univers carcéral. Mais ce faisant, il détruit son conjoint juif. Il signe littéralement son arrêt de mort. Il ne peut y avoir de dissimilation que volontaire, mais celle-ci implique crime prémédité et ralliement à la logique racialiste nazie. La seule attitude possible pour ces couples est une résistance farouche à toute tentation antisémite, en même temps, et paradoxalement, qu'une reconnaissance implicite de la nouvelle logique - pour la pervertir, la dépasser et la transformer en solidarité. Nier symboliquement la différence, refuser d'entrer dans le jeu d'une culpabilisation du conjoint par qui le malheur est arrivé, mais l'admettre concrètement afin de survivre, telle fut l'épreuve infligée à ces couples sur fond d'injures, de danger, de menaces de maladie et de mort. Nous voudrions insister sur un dernier point. Dans le Judenhaus, le conjoint aryen est constamment soumis à une telle violence qu'il peut être tenté de se voir l'otage du conjoint non aryen alors qu'en fait ils sont l'un et l'autre otages d'une même barbarie. Échapper à ce piège demandait une force de caractère, une ténacité qui n'est sans doute pas à la portée de n'importe qui. Ce qui se joue durant ces années est donc une lutte permanente de ces êtres pour dépasser la pseudo hiérarchie racialiste qu'on veut leur faire admettre et réinstaurer un ordre respectant l'unicité du genre humain. Les réactions qui transparaissent à travers les Carnets, c'est l'histoire de cette lutte, avec les hauts et les bas que fatalement elle implique.

L'épisode de l'altercation entre Klemperer et Rieger au sein de l'entreprise Schlüter révèle tout d'abord l'amertume engendrée par les cas où les épouses « aryennes » n'ont pas résisté à la tentation du divorce.

L'un d'entre nous fit l'éloge du dévouement [de nos femmes aryennes]. Rieger le contredit en s'exprimant avec la plus grande véhémence : il connaissait tant de cas déplorables, ces femmes ne tenaient pas le coup. - «Que le tonnerre les foudroie toutes, Omein - »

Klemperer refuse ce qu'il considère comme une généralisation aussi injuste que hâtive et se fâche. Plus tard, ils se réconcilient et Rieger lui confie :

Ce n'est pas vous que j'avais en tête, je ne parlais que de cas "anciens", que je connais, je pensais en particulier à la femme de Steinberg ${ }^{20}$. d'une action d'envergure contre des privilégiés devenus veufs ou dont la femme avait obtenu le divorce ${ }^{21}$. 
Deux autres cas de femmes aryennes, Frau Steinitz et Frau Kreidl senior méritent d'être mentionnés, dans la mesure où ils illustrent bien la tentation, le piège du refuge dans la différence. Concernant la première, Klemperer se fait l'écho des propos du mari :

Steinitz se plaignait de sa femme (aryenne, vingt ans plus jeune que lui), elle tempête, l'injurie, lui dit qu'il est son malheur, mais elle ne veut pas divorcer sinon sa situation financière serait trop mauvaise, mais par ailleurs, « ajoute-t-il », elle est aux petits soins pour lui, bref, une hystérique ${ }^{22}$.

Un mois plus tard, Klemperer précise : « Elle est aryenne et s'imagine courir à sa perte par sa faute à lui ${ }^{23}$ ».

Quant à Frau Kreidl senior, son cas est évoqué à l'occasion d'un incident qu'il rapporte en ces termes: "Nouvelle interdiction aux juifs de fréquenter le Grand Jardin et les autres parcs. Katz nous l'apporte pour signature... Il a une prédilection pour Eva. Pour la raison qu'elle ne s'est pas scandalisée de s'être vue attribuer une carte pour juif (Judenkarte) qui lui avait été envoyée par erreur à la place de la carte «aryenne » qui lui revient. » C'est alors qu'intervient Frau Kreidl :

Frau Kreidl senior est aussi «aryenne », et remplie d'amertume à devoir croupir dans le bourbier juif. Dans le cas d'Eva, elle a fait une scène à propos du J. Elle dit : «qu'est ce que j'en ai à faire? ». Elle devrait divorcer, dit Katz ${ }^{24}$.

Quelques semaines plus tard, Klemperer revient sur elle:

À propos de cette femme raide et froide («aryenne»), j'ai l'impression qu'une heure après le décès de son mari, elle demanderait à adhérer au parti nazi ${ }^{25}$.

Jugement dur, sans doute trop abrupt, mais qui apporte un éclairage particulièrement cru sur l'ambiance du «Judenhaus », dès lors que l'un ou l'autre s'identifie un tant soit peu au nouvel ordre racial.

Si l'on en croit les Carnets, il s'agit là en fait de réactions isolées, par rapport aux manifestations de solidarité, de compassion entre conjoints. Ainsi Frau Eisenmann évoquant son mari «négociant en charbon réputé et fortuné » devenu ajusteur chez Zeiss-Ikon et confessant outre son angoisse pour lui et leurs enfants, son chagrin profond:

Lorsque, il y a deux ans, j'ai vu mon mari pour la première fois en ouvrier, rampant couvert de graisse sous les machines, ... j'ai tant pleuré26.

Ainsi Frau Jacobi dont Klemperer affirme que, grâce à son énergie, elle a, semble-t-il, sauvé la vie de son mari. Il doit subir d'urgence une opération du cerveau. La Gestapo s'oppose à son transfert à Berlin. À l'Hôpital de Dresde il n'y a pas de chambre seule disponible. Or la salle commune est interdite aux juifs. Refus d'opérer. «Alors, raconte Klemperer, Frau Jacobi, l'aryenne, a forcé l'entrée du médecin-chef et lui a dit, ulcérée : "Vous allez donc laisser mourir mon mari parce qu'il est juif». Le médecin-chef prend alors contact avec la Gestapo, se bat pour obtenir l'autorisation d'opérer, finit par avoir gain de cause, mais pas de séjour à l'hôpital. À peine le crâne du patient était-il refermé qu'il doit regagner le Judenhaus dans un mauvais taxi ${ }^{27}$ ». Malgré cela, il survécut.

Deux autres traits caractéristiques qu'illustrent sans cesse les Carnets, sont d'une part la précarité matérielle compensée par des gestes de solidarité, d'entraide, d'autre part la détérioration progressive de la sécurité apportée par l'existence du conjoint aryen. Les exemples de solidarité et d'entraide (y compris d'ailleurs de la part de la société) sont nombreux. Ainsi Frau Voß et Frau Breit utilisent la «Kleiderkarte» d'Eva pour s'acheter des vêtements ${ }^{28}$. Klemperer note même un an plus tard qu'Eva n'a utilisé pour elle que 11 points sur les 250 disponibles. «Tout le reste est allé à des Non aryens ${ }^{29}$. ». 


\section{Le cas Bein :}

Quant à la détérioration progressive de la sécurité du conjoint juif, elle tient à deux éléments dont les Carnets sont le reflet. Le premier, c'est que fréquents sont les cas où la moindre imprudence, verbale, épistolaire ou autre de la part du conjoint juif lève, sauf de rares exceptions $s^{30}$, la protection dont il bénéficiait par son conjoint aryen. Deux exemples à ce sujet.

Femme aryenne, le mari quinquagénaire juif, le fils 18 ans, "Mischling » élevé dans la religion juive. Père et fils arrêtés il y a quelques semaines, pour avoir à ce que l'on prétend livré une trop faible quantité de textiles, transportés il y a quelques jours dans un camp de concentration à Mauthausen à la frontière autrichienne, hier " fusillés lors d'une tentative de fuite ${ }^{31}$ ».

Et le cas du cousin de Neumark:

Couple privilégié.; vit, pour des raisons économiques séparé de sa femme et de son fils Mischling d'éducation aryenne, 18 ans, dans la mesure où sa femme dirige une entreprise. Ecrit à son fils "vraisemblablement quelque chose d'imprudent. » La lettre tombe, on ne sait comment, dans les mains de la Gestapo. Le père est arrêté, trois jours plus tard, tombe la nouvelle de sa mort ${ }^{32}$.

Le second élément, c'est que la règle générale voulant qu'on ne sépare pas un couple mixte par la force, s'applique ou ne s'applique pas. À partir de 1942, plus rien n'est sûr. Klemperer apprend par Reichenbach qu'à Berlin des couples ont été séparés par la force (auseinandergerissen), et se dit alors : «Ce qui se déroule aujourd'hui à Berlin, se produira demain chez nous à Dresde ${ }^{33}$. "

L'insécurité grandissante accentue le désespoir, diminue la capacité de résistance psychique et augmente l'autodestruction. Le suicide du Dr Korn est en ce sens révélateur. Klemperer note à son sujet :

«Nouvelle perquisition, nouveau suicide. Un médecin, le Dr Korn, juif catholique, femme aryenne. La femme aryenne a été rouée de coups, le mari doit se présenter le lendemain à la Gestapo. Suicide dans la nuit. » Déclaration du commando mobile [de la Gestapo] : « Nous ferons en sorte qu'aucun de vous n'en réchappe vivant ${ }^{34}$. »

À la différence du suicide, qui est un geste a priori délibéré, le sort du conjoint juif dépend aussi de la santé du conjoint aryen. S'il vient à mourir, la déportation intervient sans attendre. Klemperer rapporte entre autre le cas Garnmann et le cas Heim. Frau Garnmann meurt subitement, le mari a les plus grandes peines à obtenir l'autorisation de pénétrer dans le cimetière pour assister à l'enterrement de sa femme, ensuite, déportation à Theresienstadt. Quant à Heim, sa femme meurt d'un cancer le 27 février 1944 : il doit immédiatement « faire sa valise » et est acheminé vers Theresienstadt ${ }^{35}$.

Ces quelques exemples ne peuvent naturellement prétendre à la moindre exhaustivité. Ils montrent en tout cas, et sans vouloir établir de hiérarchie entre les différentes formes de martyre endurées par les innombrables victimes du nazisme, que ces couples, ont été tous confrontés à une torture morale particulièrement sophistiquée, à une épreuve redoutable dont même un amour sans faille, même une fidélité indéfectible, et une santé de fer, ne pouvaient garantir qu'elle serait surmontée. L'épée de Damoclès, pour eux, n'a pas été une vaine formule. 
44 Klemperer, son itinéraire à travers ce long calvaire, sa spécificité, telle qu'elle apparaît au fil des pages. du nouvel ordre racial. Leur identité de couple est progressivement remise en question. Victor vit comme une succession de cauchemars la dissimilation dont il est l'objet, sa relégation à un statut social inférieur, tandis que pour Eva, qu'aucune mesure ne frappe d'emblée directement, c'est le sort fait à son mari et les premières conséquences liées à la dissimilation qui l'accablent. Marginalisation, déclassement social, prolétarisation économique, solitude surtout, telles sont les notations les plus récurrentes des Carnets : «isolement, Eva déprimée» (3.12.33) ; «vie de journalier» (10.2.35) ; «nous nous enterrons littéralement ici, comme dans une tranchée» (21.7.35); "nous sommes toujours plus solitaires » (24.4.36); « nous sommes totalement isolés » (28.4.36) ; " nous sommes en fait complètement prolétarisés » (18.7.36) ; « la solitude est toujours plus pesante » (28.12.37) ; "solitude absolue » (23.2.37), avec ce bémol, cependant qui va devenir l'un des leitmotive des Carnets: "Dans notre profonde solitude, nous nous sentons encore plus proches que dans les années antérieures» (27.3.37). Le 3.12.38 se concrétise soudain la différence de statut « racial» entre les deux conjoints. Victor reçoit son « Israël », sa nouvelle carte d'identité avec « photo de criminel », Eva reçoit son attestation d'appartenance à la race aryenne («Ariernachweiss »). Ils vont faire progressivement l'apprentissage de cette nouvelle révision de leur statut respectif. Le début de la spoliation des citoyens d'origine juive nous le montre incapable de réagir en fonction de la nouvelle logique. Le 7.4.39, il déclare être son bien propre des bijoux appartenant à Eva, ce qui équivaut à les perdre alors que sa qualité d'aryenne eut permis de les sauver. Il qualifie son comportement d'insensé. Le premier avril 1939, ils reçoivent l'ordre de quitter leur maison: «Vous avez droit à une pièce. Comme votre femme est aryenne, peut-être vous accordera-t-on deux pièces ${ }^{36}$ ». Ils voient alors s'écrouler leur univers familier, le jardin aménagé par Eva détruit par les locataires.

L'installation dans le Judenhaus, le 26 mai 1940, paradoxalement, les resocialise. La communauté est certes artificielle, mais elle semble provoquer le début aussi du recours à la différence de statut pour, partiellement au moins, la dépasser. Victor qui a horreur de montrer dans les magasins sa carte de juif - « il y a toujours à côté de moi des gens qui voient le $\mathrm{J}$ » - utilise dès que possible la carte « aryenne » d'Eva ${ }^{37}$.

La solitude dans la promiscuité leur pèse. Premier heurt entre eux : « l'interdiction des parcs et jardins prive Eva de longues promenades » la seule chose qui lui restait encore. Ils continuent à découvrir leur nouvelle différence. Exemple : le café. 50 grammes pour les aryens, pour les juifs rien. Ils boivent ensemble chacun une tasse. Victor : «c'était presque comique ${ }^{38}$. »

Eva s'ennuie, lui fait des reproches. Reproche majeur, qui ouvre une période nouvelle dans leurs relations: elle se plaint de n'avoir pas été entendue à propos de la construction de la maison à Dölzschen. Tout est venu trop tard. Et surtout : Victor n'a pas mis la maison à son nom alors qu'il en était encore temps. C'est là le début d'un processus de remise en cause des fondements du couple, Eva accusant ouvertement Victor de lui avoir gâché sa vie. "Je suis blessé d'entendre Eva dire qu'elle vit depuis des années à côté de sa vie $»^{39}$. Face à l'épreuve, les contentieux anciens refont surface, mais ne conduisent pas à une destruction. En fait, le couple se reconstruit sur de 
nouvelles bases. En a-t-il conscience? Ce n'est pas évident. En tout cas, Victor note le 6 juillet 1941 :

Peut-être que ce qu'il y avait de bon dans cette période de souffrance, c'est que nous avons acquis une conscience nouvelle de nos affinités, de notre bonheur, de l'absolue insignifiance de toute chose en dehors de notre vie ensemble ${ }^{40}$.

L'épisode marquant de la prison est l'occasion pour lui d'un véritable examen de conscience. Il s'aperçoit à quel point il aime sa femme, se remet partiellement en question, reconnaît qu'il s'est toujours accordé la prééminence au sein du couple. Le métier d'Eva, musicienne, n'a jamais été pour lui qu'un « agréable à côtéte ${ }^{41}$.

Nous apprenons quel fut l'itinéraire d'Eva. Accident et maladie la privent de son métier ; elle souhaite une villa et un jardin en guise d'occupation et de compensation. Mais cette perspective lui est refusée par un mari pétri d'angoisse: angoisse de s'installer, angoisse des charges financières que cela implique. Confession unique, dans toute l'œuvre, qui a en outre l'avantage de révéler une personnalité à la fois dépendante et profondément égocentrique ${ }^{42}$. Nous y reviendrons.

51 Avec l'avalanche des mesures de dissimilation et surtout le port de l'étoile juive, s'accentue la dépendance matérielle et affective de Victor par rapport à Eva. Elle devient peu à peu le seul lien avec le monde extérieur. Son ennui va grandissant, mais notons-le au passage, alors qu'elle nous est constamment présentée comme "déprimée ", souffrante, malade, s'impose peu à peu l'image d'une femme assurant l'essentiel de la vie matérielle, généreuse, solidaire, capable d'une exceptionnelle résistance face à l'adversité. Elle affronte la Gestapo avec courage et dignité : "Ces brutes sont des lâches ${ }^{43}$ ».

52 L'épisode de la mort de leur chat illustre la cruauté gratuite des mesures imposées par un pouvoir sadique et délirant. Dans le cas présent, la condition d'Aryenne ne joue pas. Tous les animaux domestiques sont voués à la mort. Qu'Eva supporte mal ce deuil est d'autant plus compréhensible que ce chat-fétiche, ersatz d'enfants qu'ils n'ont pas, était devenu le symbole de leur résistance ${ }^{44}$.

53 À cette occasion, elle manifeste un nouvel accès d'amertume par rapport à son mari. Mais les difficultés s'amoncelant, l'esprit de solidarité, de résistance reprend le dessus, même si sa déchéance physique l'éprouve parfois durement. Son importance croît. Son rôle d'intermédiaire entre la communauté du Judenhaus et l'extérieur également. Elle se heurte à Victor sur la vente éventuelle de la maison, et obtient apparemment gain de cause après avoir encouragé son mari à ne pas se laisser intimider par les pressions qu'on exerce sur lui pour le pousser à vendre. Mais à cette occasion, transparaît dans la discussion tout l'arrière plan morbide de leur situation. Ne lui dit-elle pas en effet :

Pour parler à ta manière : je serai donc responsable si tu aboutissais en camp de concentration? Comme si ces gens-là avaient besoin d'un prétexte pour assassiner, et justement ce prétexte-làa ${ }^{45}$.

En dépit de ces affrontement, la capacité de résistance d'Eva s'affirme au fur et à mesure que se concrétise la dépendance quasi totale où se trouve son mari par rapport à elle. «Je ne suis rien sans Eva ", écrit-il fin 1943, et s'aperçoit que vivre sans elle lui est impensable ${ }^{46}$.

De plus en plus, s'affirme chez elle un stoïcisme sans faille grâce auquel ils parviendront, la chance aidant, à sortir vivants de ces douze années d'épreuves.

Sans nul doute, l'amour, la qualité du lien affectif ont été déterminants dans la capacité de ces couples à résister à la tentation d'un retour à la «norme " par le sacrifice de 
l'autre. Dans le cas précis d'Eva et de Victor, il n'est guère possible de faire abstraction du profil psychologique de deux êtres fondamentalement différents, mais l'un comme l'autre, paradoxalement assez bien armés a priori pour affronter ce type d'épreuve. Égocentrique, enfermé dans une névrose qui peu à peu se révèle au lecteur - tendance à l'hypochondrie d'une part, recours obsessionnel à l'écriture d'autre part comme unique antidote à l'angoisse (d'où la compulsion de répétition permanente que traduit le fleuve des Carnets, d'où l'horreur de la prison où, privé d'un crayon et d'un papier il ne vit plus, où retrouvant l'un et l'autre, il se remet à vivre ${ }^{47}$ ), Victor ne vit rien d'autre durant ces douze années que ce que psychiquement, il a toujours vécu: angoisse, refoulement, hantise de la maladie et de la mort ${ }^{48}$. Le discours est étonnamment le même du début à la fin de la période, y compris lorsque son statut de paria s'effondre et que, après mai 1945, il devient une sorte de héros.

Quant à Eva, qui apparemment s'est accommodée d'un tel homme durant tant d'années, elle apparaît finalement comme un être ayant pu, paradoxalement, réévaluer sa raison d'être, retrouver une vie sociale, rééquilibrer son couple en sa faveur à travers l'épreuve endurée. Elle se retrouve peu à peu assumant pour son mari d'abord, pour les autres ensuite, dans la situation d'une mère responsable de son mari-enfant, et tenue de résister jusqu'au bout à ses accès d'angoisse.

Ces réflexions finales n'ôtent en rien leur dimension héroïque à la volonté de témoigner «jusqu'au bout» de Victor comme celle de tenir «jusqu'au bout» d'Eva. Elle le relativise simplement, et par la même, lui confère toute sa valeur humaine.

\section{NOTES}

1. . Victor Klemperer, LTI, Reclams Universalbibliothek, Bd 278, Leipzig, p. 14.

2. Ibidem.

3. . Victor Klemperer, Ich will Zeugnis ablegen bis zum letzten, Tagebücher 1933-1941, 1942-1945, Aufbau-Verlag, Berlin 1995.

4. . Carnets I, 15, 82, 111, 438 (Mein Deutschtum).

5. . Janine Chasseguet-Smirgel, «Le corps de Sade» in: Corps création. Entre lettres et psychanalyse, sous la direction de Jean Guillaumin, Lyon, 1980, p. 169-182.

6. . Carnets, I, 16.

7. . Cette adjonction anticipe le décret d'application de la loi sur la citoyenneté (Reischbürgergesetz) de septembre 1935, qui, le 14 novembre 1935, précisera enfin ce qu'il faut entendre par «non aryen ».

8. . Carnets, I, 468.

9. Wilhem Rödiger, Geschichte, Ziel, Stoff und Weg, Leipzig 1934, p. 24.

10. . Deutsche Volksgesundheit, 1.1.1935. Cité d'après Kennzeichen J., Dokumente, Berichte zur Geschichte der Verbrechen des Hitlerfaschismus an den deutschen Juden 1933-1945, Berlin 1966, doc. VIII /1 p. 83-84.

11. . Juden unterm Hakenkreuz, v. Klaus Drobisch, Rudi Goguel, Werner Müller unter Mitwirkung von Horst Dohle, Berlin (Est), 1973, p. 210.

12. . Carnets, I, 496. 
13. . Medizin ohne Menschlichkeit, v. A. Mitscherlich U. F. Mielke, Francfort/Main, 1960, p. 212.

14. . Carnets I, 121.

15. Juden unterm Hakenkreuz, op. cit., p. 347-48.

16. . Ibidem, p. 360.

17. . Carnets II, 116.

18. . Ibid. I, 577.

19. . Ibid. I, 533.

20. . Ibid. II, 475 .

21. . Ibid. II, 471.

22. . Ibid. II, 32.

23. . Ibid. II, 67.

24. . Ibid. II, 536.

25. . Ibid. II, 542.

26. . Ibid. I, 267.

27. . Ibid. II, 341.

28. . Ibid. I, 551 et I, 671-72.

29. . Ibid. I, 656.

30. . Ibid. II, 257.

31. . Ibid. II, 252.

32. . Ibidem.

33. . Ibid. II, 266.

34. . Ibid. II, 76.

35. . Ibid. II, 456 et 491.

36. . Ibid. I, 503.

37. . Ibid. I, 536.

38. . Ibid. I, 559.

39. . Ibid. I, 598.

40. . Ibid. I, 601.

41. . Ibid. I, 634.

42. . Ibid. I, 634.

43. . Ibid. II, 119-20.

44. . Ibid. II, 85.

45. . Ibid. II, 310.

46. . Ibid. II, 445 .

47. . Ibid. I, 638 et II, 184.

48. . Ibid. I, 28, I, 72 et II, 184.

\section{RÉSUMÉS}

L'objet de cette contribution est l'étude d'un cas de «Mischehe » («mariage mixte ») sous le Troisième Reich. Le point de départ est la racialisation du concept de Mischehe. Celui-ci désignait en Allemagne depuis des lustres une union entre personnes d'appartenance religieuse différente. Après 1933, le terme sert à désigner un couple des "races différentes ", en l'occurrence essentiellement Allemands « aryens » et Allemands d'origine juive. 
Suit alors l'étude de la politique nazie à l'égard des « Mischehen » et de son évolution.

Le grand intérêt du Journal de Victor Klemperer, qui constitue avec sa femme Eva une " Mischehe », est qu'il permet de retracer jour après jour les formes et les conséquences de cette politique, à la fois sur le plan pratique et sur le plan psychologique. Nous pénétrons ainsi dans la vie quotidienne, voire dans l'intimité d'un couple, Victor et Eva, qui révèle ses défauts et ses qualités à travers cette douloureuse expérience vécue et souligne le caractère spécifique de chacun.

Mais le témoignage de Victor Klemperer ne se limite pas à son propre couple.. Il se fait l'écho de la manière dont d'autres «Mischehen» (57 en tout), rencontrées dans les sinistres «Judenhäuser, ces « camps de concentration haut de gamme» (Klemperer), ont tant bien que mal affronté l'épreuve. Les épouses " aryennes » ont dans leur ensemble prouvé une étonnante capacité à résister, au point que leur attitude a été qualifiée par l'auteur du Journal d'héroïsme transcendant tous les autres.

Thema dieses Vortrags ist die Untersuchung des Schicksals einer « Mischehe » im Dritten Reich. Den Ausgangspunkt bildet die rassische Pervertierung des Mischehe-Begriffes: dieser bezeichnete seit eh und je in Deutschland eine Eheschliessung von Partnern verschiedener Religions- oder Konfessionszugehörigkeit ; nach 1933 kennzeichnet er eine Ehe, in der sich « zwei unterschiedliche Rassen miteinander vermischen", wobei hauptsächlich an die eheliche Verbindung zwischen « Ariern » und deutschen Bürgern jüdischer Herkunft gedacht wird.

Unsere Untersuchung beginnt mit der Darlegung der nazistischen Politik den « Mischehen » gegenüber, die manche Wandlungen erfuhr.

Besonders interessant ist in dieser Hinsicht das Tagebuch von Victor Klemperer, der mit seiner « arischen » Frau Eva eben eine sog. Mischehe bildet: wir verfolgen Tag für Tag die Formen und Konsequenzen jener Politik, und zwar sowohl auf praktischer als auf psychologischer Ebene. Wir dringen dadurch ins Alltagleben eines Ehepaares, Victor und Eva, das seine Schwächen und positiven Eigenschaften jener schmerzhaften Erfahrung gegenüber enthüllt: der spezifische Charakter jedes einzelnen wird damit ans Licht gebracht.

Aber die Zeugnisablegung von Victor Klemperer ist nicht bloss die persönliche Geschichte eines Ehepaares. Sie dient als Spiegelbild der Art und Weise, wie andere « Mischehen » (57 insgesamt), in der grauenhaften Atmosphäre des «Judenhauses » versammelt, solche Zumutung so gut wie möglich ertragen haben. Die " arischen» Frauen haben im grossen ganzen eine erstaunliche Fähigkeit zum Widerstand gezeigt, so dass Victor Klemperer ihre Haltung später als « Heroismus über jeglichen Heldentum » bezeichnen konnte.

\section{AUTEUR}

\section{DIDIER HERLEM}

Université Charles-de-Gaulle - Lille 3 